\title{
A High Order WENO-Z Finite Difference Based Particle-Source-in-Cell Method for Computation of Particle-Laden Flows with Shocks
}

\author{
Gustaaf B. Jacobs * Wai-Sun Don ${ }^{\dagger}$
}

June 2,2008

\begin{abstract}
A high order particle-source-in-cell (PSIC) algorithm is presented for the computation of the interaction between shocks, small scale structures, and liquid and/or solid particles in high-speed engineering applications. The improved high order finite difference weighted essentially non-oscillatory (WENO-Z) method for solution of the hyperbolic conservation laws that govern the shocked carrier gas flow, lies at the heart of the algorithm. Finite sized particles are modeled as points and are traced in the Lagrangian frame. The physical coupling of particles in the Lagrangian frame and the gas in the Eulerian frame through momentum and energy exchange, is numerically treated through high order interpolation and weighing. The centered high order interpolation of the fluid properties to the particle location is shown to lead to numerical instability in shocked flow. An essentially non-oscillatory interpolation (ENO) scheme is devised for the coupling that improves stability. The ENO based algorithm is shown to be numerically stable and to accurately capture shocks, small flow features and particle dispersion. Both the carrier gas and the particles are updated in time without splitting with a third order Runge-Kutta TVD method. One and two dimensional computations of a shock moving into a particle cloud demonstrates the characteristics of the WENO-Z based PSIC method (PSIC/WENO-Z). The PSIC/WENO-Z computations are not only in excellent agreement with the numerical simulations with a third order Rusanov based PSIC and physical experiments in [Boiko et al. Shock Waves 7, 1997], but also show a significant improvement in the resolution of small scale structures. In two dimensional simulations of the Mach 3 shock moving into forty thousand bronze particles arranged in the shape of a rectangle, the long time accuracy of the high order method is demonstrated. The fifth order PSIC/WENO-Z method with the fifth order ENO interpolation scheme improves the small scale structure resolution over the third order PSIC/WENO-Z method with a second order central interpolation scheme. Preliminary analysis of the particle interaction with the flow structures shows that sharp particle material arms form on the side of the rectangular shape. The arms initially shield the particles from the accelerated flow behind the shock. A reflected compression wave, however, reshocks the particle arm from the shielded area and mixes the particles.
\end{abstract}

\section{Keywords}

Particle-Source-In-Cell, WENO, Particle-Mesh, Eulerian-Lagrangian, Shock-Particle-Laden, Hyperbolic Conservation Laws

\footnotetext{
*Department of Aerospace Engineering \& Engineering Mechanics, San Diego State University, San Diego, CA 92182 E-Mail: gjacobs@mail.sdsu.edu

${ }^{\dagger}$ Division of Applied Mathematics, Brown University, Providence, Rhode Island 02912. E-Mail: wsdon@dam.brown.edu
} 


\section{Introduction}

High-speed gas flows encountered in supersonic aircraft, hypersonic space vehicles, gas turbines and explosions are commonly modeled through the nonlinear hyperbolic conservation laws in the form

$$
\frac{\partial \mathbf{Q}}{\partial t}+\nabla \cdot \mathbf{F}(\mathbf{Q})=0
$$

where $\mathbf{Q}$ is a vector of conservative variables and $\mathbf{F}$ is the flux vector. Shocks are typical in these high-speed flows and can appear as finite time singularity solutions in the conservation laws.

For the capturing of shock solutions in numerical approximations of (1) many nonlinear shock capturing schemes have been devised, e.g. TVD, PPM, ENO, CENO and WENO schemes. Among these schemes, the high order, high resolution finite difference Weighted Essentially Non-Oscillatory schemes (WENO) $[1,2]$ have proven to be very effective in capturing the fine scale and delicate structures of the physical phenomena involving large gradients and shocks. The classical WENO schemes owe their success to the use of a dynamic set of stencils, where a nonlinear convex combination of lower order polynomials adapts either to a higher order approximation at smooth parts of the solution, or to an upwind spatial discretization that avoids interpolation across discontinuities and provides the necessary dissipation for shock capturing. The nonlinear coefficients of the convex combination, hereafter referred to as weights, are based on the local smoothness indicators, which measure the sum of the normalized squares of the scaled $L^{2}$ norms of all derivatives of the lower order polynomials [2]. An essentially zero weight is assigned to those lower order polynomials whose underlining stencils contain high gradients and/or shocks, yielding an essentially non-oscillatory solution at and near discontinuities. At smooth parts of the solution, higher order is achieved through the mimicking of the central upwinding scheme of maximum order, when all smoothness indicators are about the same size. The classical WENO schemes are designed based on the successful class of high order schemes called the essentially non-oscillatory, or ENO schemes of Harten et al. [3, 4, 5]. The first classical WENO scheme was introduced by Liu et al. in their pioneering paper [6], in which a third order accurate finite volume WENO scheme was designed. In 1996, Jiang and Shu [2] provided a general framework to construct arbitrary order accurate finite difference WENO schemes, which are more efficient for multi-dimensional calculations. Higher order finite difference classical WENO schemes are designed in [1]. Improvement of the classical WENO scheme, namely the mapped WENO scheme [7] and WENO-Z scheme [8], was also derived. In this study, the high order WENO-Z scheme is employed. In comparison to classical WENO schemes, the WENO-Z method has a lower dissipation in combination with a proven formal order of accuracy [8]. As a result the method captures shock in a physically sharp manner, while the high order and high resolution nature of the scheme ensures an efficient and accurate resolution of small scales in smooth regions.

In many high-speed flow applications, shocks interact with solid or liquid particles. For example, debris interacts with shocks and gas turbulence in dust explosions. In lithotripsy, kidney stones are broken into smaller kidney stone particles by means of shocks. Fuel droplets interact with a chemically reacting gas containing shock waves in high-speed combustors. When the flow containing shocks is turbulent, the scale range of the continuum flow is enormous. The mutual exchange of momentum and energy of particles and the continuum flow results in even more complex, multi-scale physics. The tremendous complexity of the problem leaves many physical phenomenon less understood.

The direct analysis requires the computation of the complete flow over each particle, the tracking of individual solid or liquid complex particle boundaries along their paths, and the tracking of shock waves in the moving frame. These individual computational components are difficult to resolve and currently barely within reach even with the latest advances of computational technologies. The combined interaction between flows with shocks and particles has an immense complexity, scale range and size, that can currently potentially be analyzed only in highly idealized situation with a few particles.

Simplified models for the particle and the particle interactions with the shock containing carrier gas are required to handle more realistic situation in applications of shocked particle-laden flows. The point particle modeling in the Particle-Source-in-Cell (PSIC) method introduced by Crowe et al. [9] provides such a model that enables larger scale analysis. The PSIC method is a particle-mesh type algorithm, that is 
also commonly used for simulation of plasma [10]. In particle-mesh type methods, the continuum model is approximated on a static mesh, while the particle dynamics are traced along their path in a Lagrangian frame. The static mesh solution and the dynamic particle mesh solution are subsequently coupled through interpolation. In the PSIC method, particles are assumed to have a single point contribution. Point particles are carried by the carrier gas flow that is governed by a continuum equation, which in this study are the Euler equations governing the gas dynamics in an Eulerian frame. The influence of the particles on the carrier gas flow is modeled through point sources and appears as source terms in the continuum equations. The point modeling permits the simulation of a large number of particles and provides sufficient resolution and accuracy to model the particle-laden flows. Particle-mesh is the only method that facilitates affordable computations of real geometries, while accurately representing individual particle dynamics. The Cloud-In-Cell (CIC) [11] modeling enhances the computational feasibility by decreasing the modeling level of the particle. Rather than modeling a single particle, a cloud of particles is modeled as point contribution in CIC. The individual cloud dynamics are traced in the Lagrangian frame like in the single particle model. While with the cloud modeling some of the particle detail is less, it can be shown to be sufficiently accurate for most practical applications [11]. In this study, we shall refer to particle clouds as particles unless specified otherwise.

The particle-mesh method typically relies on relatively simple, coarse grids and/or low order numerical schemes (typically first or second order schemes). This limits the treatment of realistic problems with a large range of active temporally and spatial scales. Dispersion, diffusion and aliasing errors are well-known to plague the spatial accuracy of the solution [10]. To reduce these errors, high order high resolution numerical algorithms are essential for efficient long time simulation of the shock-particle-laden flow [13]. In Jacobs and Hesthaven [14, 15], a high-order based particle-mesh method for simulation of plasma was shown to reduce these errors. The high-order, high-resolution WENO methods were also shown to capture small scales structures more accurately and efficiently than the lower order schemes in the long time simulation of the Richtmeyer-Meshkov Instability [16].

The main purpose of this study is to extend the advantages of high order methods to a high order PSIC method based on the high-order, high-resolution WENO-Z scheme (PSIC/WENO-Z). We propose a high order ENO interpolation from the carrier flow to the particles and a high order weighing of the momentum and energy from the particle to the carrier flow, yielding a high order solution of particle-laden gas flow with shocks in the Eulerian-Lagrangian frame. We also identify future improvements and extension of the algorithm to more realistic applications.

We first validate the the PSIC/WENO-Z method and verify accuracy and efficiency improvements with the one dimensional numerical lower PSIC simulation and experiments in Boiko et al. [12]. We then present the two dimensional simulation of shock interaction with $40 \mathrm{~K}$ bronze particle arranged in a rectangular shape in front of a Mach 3 shock. The setup is the same as the one used in the numerical experiment with PSIC method based on a third order Rusanov scheme in [13] except that the flow is assumed to be periodical in the $y$ direction. At early times the large and small scales structures are similar to the results in [13] . We show that the capturing of small scale with a fifth order PSIC/WENO-Z method is superior to the capturing with a third order PSIC/WENO-Z method. We illustrate long time accuracy of high order PSIC/WENO-Z with new results of the shock-particle interaction for long time evolution of the particle-laden flow field in a large physical domain. The behavior of the flow and particle trajectories shows a strong nonlinear interaction and coupling of the carrier flow and the particles and warrants a further detailed numerical and experimental investigation.

This paper is organized as follows: In section 2, the physical model of the carrier flow and the particles are presented. In section 3, we give a brief description of the improved fifth order weighted essentially nonoscillatory finite difference WENO-Z scheme for hyperbolic conservation laws. In section 4 the coupling of the carrier flow in the Eulerian frame and the particle in the Lagrangian frame is discussed in detail. We present the high order ENO interpolation scheme that is used to interpolate the gas properties from the Eulerian frame to the particle in the Lagrangian frame. The high order weighing for exchange of momentum and energy from the particle in the Lagrangian frame to the carrier gas at a grid point in the Eulerian frame are also given. Numerical experiments with the PSIC/WENO-Z method are discussed in section 5. The high order PSIC/WENO-Z method is validated against existing one dimensional numerical results 
and experimental data. The long time accuracy is also demonstrated in long time simulations of the two dimensional shock interaction with $40 \mathrm{~K}$ bronze particle. Conclusions and direction of future research are reserved for the final section 6 .

\section{The Physical Model and Particle-Source-In-Cell Method}

In the particle-source-in-cell (PSIC) method the Eulerian continuum equations are solved for the carrier flow in the Eulerian frame, while particles are traced along in the Lagrangian frame. In the following, we present the coupled system of Euler equations that govern the gas flow and kinematic equations that govern the particle motion. We shall denote the subscript $p$ for the particle variables and $f$ for the gas variables at the particle position. Variables without subscript refer to the gas variables unless specified otherwise.

\subsection{Euler equation in the Eulerian frame}

The governing equations for the carrier flow are the two-dimensional Euler equations in Cartesian coordinates given by:

$$
\mathbf{Q}_{t}+\mathbf{F}_{x}+\mathbf{G}_{y}=\mathbf{S}
$$

where

$$
\begin{aligned}
\mathbf{Q} & =(\rho, \rho u, \rho v, E)^{T}, \\
\mathbf{F} & =\left(\rho u, \rho u^{2}+P, \rho u v,(E+P) u\right)^{T}, \\
\mathbf{G} & =\left(\rho v, \rho u v, \rho v^{2}+P,(E+P) v\right)^{T},
\end{aligned}
$$

and

$$
P=(\gamma-1)\left(E-\frac{1}{2} \rho\left(u^{2}+v^{2}\right)\right), \quad \gamma=1.4
$$

The equation of state closes the system of equations

$$
T=\frac{\gamma P M^{2}}{\rho}
$$

where $M=U / \sqrt{\gamma R T}$ is a reference Mach number determined with the reference velocity, $U$ and reference

temperature, $T$. The source term, $\mathbf{S}$, accounts for the effect of the particles on the carrier gas and will be discussed in more detail below.

\subsection{Particle equation in the Lagrangian frame}

Particles are tracked individually in the Lagrangian frame. The kinematic equation describing the particle's position $\vec{x}_{p}$, is given as

$$
\frac{d \vec{x}_{p}}{d t}=\vec{v}_{p}
$$

where $\vec{v}_{p}$ is the particle velocity vector.

The particles' acceleration is governed by Newton's second law forced by the drag on the particle. With particles assumed spherical, we take the drag as a combination of the Stokes drag corrected for high Reynolds and Mach number and the pressure drag leading to the following equations governing the particle velocity $[12]$

$$
\frac{d \vec{v}_{p}}{d t}=f_{1}\left(\frac{\vec{v}_{f}-\vec{v}_{p}}{\tau_{p}}\right)-\left.\frac{1}{\rho_{p}} \nabla P\right|_{f}
$$


where $\vec{v}_{f}$ is the velocity of the gas at the particle position, and $\rho_{p}$ the particle density. The first term on the right hand side describes the particle acceleration resulting from the velocity difference between the particle and the gas. The second term in the right hand side of (7) represents the particle acceleration induced by the pressure gradient in the carrier flow at the particle position. The particle time constant $\tau_{p}=\operatorname{Re} d_{p}^{2} \rho_{p} / 18$, where $d_{p}$ is the particle diameter, is a measure for the reaction time of the particle to the changes in the carrier gas. $R e=U L / \nu$ is the Reynolds number of the carrier gas flow with $L$ a reference length and $\nu$ the dynamic viscosity. In this study, we assume $R e$ large and we therefore do not model viscous effects in the governing Eulerian equations for the gas flow (2).

$f_{1}$ is an empirical correction factor [12] that yields an accurate determination within $10 \%$ of measured particle acceleration for higher relative particle Reynolds number up to $R e_{f}=\left|\vec{v}_{f}-\vec{v}_{p}\right| d_{p} / \nu=1 \times 10^{4}$ and relative particle Mach number up to $M_{f}=\left|\vec{v}_{f}-\vec{v}_{p}\right| / \sqrt{T_{f}}=1.2$ and is given by

$$
f_{1}=\frac{3}{4}\left(24+0.38 R e_{f}+4 \sqrt{R e_{f}}\right)\left(1+\exp \left[\frac{-0.43}{M_{f}^{4.67}}\right]\right) .
$$

From the first law of thermodynamics and Fourier's law for heat transfer, the equation for temperature is derived as,

$$
\frac{d T_{p}}{d t}=\frac{1}{3} \frac{N u}{\operatorname{Pr}}\left(\frac{T_{f}-T_{p}}{\tau_{p}}\right)
$$

where $\operatorname{Pr}=1.4$ is the Prandtl number, taken as its typical value for air in this paper. $N u=2+\sqrt{\operatorname{Re}_{f}} \operatorname{Pr}^{0.33}$ is the Nusselt number corrected for high Reynolds number.

\subsection{Source term $\mathrm{S}$ for the Euler equation}

Each particle generates a momentum and energy that affects the carrier flow. The volume averaged summation of all these contributions gives a continuum source contribution on the momentum and energy equation in (2) as:

$$
\begin{aligned}
\vec{S}_{m}(\vec{x}) & =\sum_{i=1}^{N_{p}} \mathbf{K}\left(\vec{x}_{p}, \vec{x}\right) \vec{W}_{m}, \\
S_{e}(\vec{x}) & =\sum_{i=1}^{N_{p}} \mathbf{K}\left(\vec{x}_{p}, \vec{x}\right)\left(\vec{W}_{m} \cdot \vec{v}_{p}+W_{e}\right),
\end{aligned}
$$

where $\mathbf{K}(x, y)=\mathbf{K}(|x-y|) / V$ is a normalized weighing function that distributes the influence of each particle onto the carrier flow. $\vec{W}_{m}=m_{p} f_{1}\left(\vec{v}_{f}-\vec{v}_{p}\right) / \tau_{p}$ and $W_{e}=m_{p}(N u /(3 P r))\left(T-T_{p}\right) / \tau_{p}$ are weigh functions describing the momentum and energy contribution of one particle, respectively. $m_{p}$ is the mass of one spherical particle which can be derived from $\tau_{p}$. $N_{p}$ is the total number of particles in an finite volume $V$. The high order normalized weighing function will be discussed in section 4 .

\section{Improved Weighted Essentially Non-Oscillatory Schemes}

In this section we briefly describe the fifth-order weighted essentially non-oscillatory conservative finite difference scheme (WENO-Z) [8] when applied to hyperbolic conservation laws as in (1). Higher order versions up to eleventh order of the WENO-Z scheme have been developed and will appear in another article. Without loss of generality, we will restrict our discussion to the one dimensional scalar case and the fifth order WENO-Z reconstruction step. The extension to a system of equations and to higher spatial dimensions is straightforward in the Cartesian coordinates.

Consider a uniform grid defined by the points $x_{i}=i \Delta x, i=0, \ldots, N$, which are also called cell centers, with cell boundaries given by $x_{i+\frac{1}{2}}=x_{i}+\frac{\Delta x}{2}$, where $\Delta x$ is the uniform grid spacing. The semi-discretized 


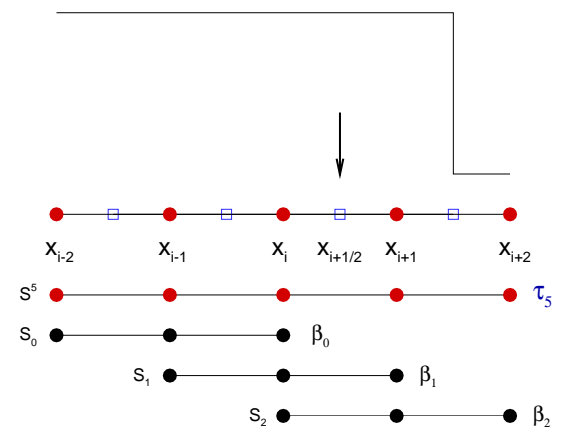

Figure 1: The computational uniform grid $x_{i}$ and the five points stencil $S^{5}$, composed of three 3-points stencils $S_{0}, S_{1}, S_{2}$, used for the fifth-order WENO reconstruction step.

form of (1), by the method of lines, yields a system of ordinary differential equations

$$
\frac{d u_{i}(t)}{d t}=-\left.\frac{\partial f}{\partial x}\right|_{x=x_{i}}, \quad i=0, \ldots, N
$$

where $u_{i}(t)$ is a numerical approximation to the point value $u\left(x_{i}, t\right)$.

A conservative finite-difference formulation for hyperbolic conservation laws requires high-order consistent numerical fluxes at the cell boundaries in order to form the flux differences across the uniformly-spaced cells. The conservative property of the spatial discretization is obtained by implicitly defining the numerical flux function $h(x)$ as

$$
f(x)=\frac{1}{\Delta x} \int_{x-\frac{\Delta x}{2}}^{x+\frac{\Delta x}{2}} h(\xi) d \xi,
$$

such that the spatial derivative in (12) is exactly expressed by a conservative finite difference formula at the cell boundaries,

$$
\frac{d u_{i}(t)}{d t}=\frac{1}{\Delta x}\left(h_{i+\frac{1}{2}}-h_{i-\frac{1}{2}}\right)
$$

where $h_{i \pm \frac{1}{2}}=h\left(x_{i \pm \frac{1}{2}}\right)$.

High order polynomial interpolations to $h_{i \pm \frac{1}{2}}$ are computed using known grid values of $f, f_{i}=f\left(x_{i}\right)$. The fifth-order WENO-Z scheme uses a 5 -points stencil, hereafter named $S^{5}$, which is subdivided into three 3-points stencils $\left\{S_{0}, S_{1}, S_{2}\right\}$, as shown in Fig. 1. The fifth-order polynomial approximation $\hat{f}_{i \pm \frac{1}{2}}=$ $h_{i \pm \frac{1}{2}}+O\left(\Delta x^{5}\right)$ is built through the convex combination of the interpolated values $f^{k}\left(x_{i \pm \frac{1}{2}}\right)$, in which $f^{k}(x)$ is the third degree polynomial below, defined in each one of the stencils $S_{k}$ :

$$
\hat{f}_{i \pm \frac{1}{2}}=\sum_{k=0}^{2} \omega_{k}^{Z} \hat{f}^{k}\left(x_{i \pm \frac{1}{2}}\right)
$$

where

$$
\hat{f}^{k}\left(x_{i+\frac{1}{2}}\right)=\hat{f}_{i+\frac{1}{2}}^{k}=\sum_{j=0}^{2} c_{k j} f_{i-k+j}, \quad i=0, \ldots, N .
$$


The $c_{k j}$ are Lagrangian interpolation coefficients (see [2]), which depend on the left-shift parameter $k=0,1,2$, but not on the values $f_{i}$.

The WENO-Z weights $\omega_{k}^{Z}$ are defined as

$$
\omega_{k}^{Z}=\frac{\alpha_{k}^{Z}}{\sum_{l=0}^{2} \alpha_{l}^{Z}}, \quad \alpha_{k}^{Z}=\frac{d_{k}}{\beta_{k}^{Z}}, \quad k=0,1,2 .
$$

The coefficients $d_{0}=\frac{3}{10}, d_{1}=\frac{3}{5}, d_{2}=\frac{1}{10}$ are called the ideal weights since they generate the central upstream fifth-order scheme for the 5-points stencil $S^{5}$. The weights $\omega_{k}^{Z}$ are a function of the smoothness indicators $\beta_{k}^{z}$, namely,

$$
\beta_{k}^{Z}=\left(1+\left(\frac{\tau_{5}}{\beta_{k}+\epsilon}\right)^{2}\right),
$$

where $\epsilon$ is a small number (typically $\epsilon=10^{-12}$ ) used to avoid the division by zero in the denominator of (17) and the classical smoothness indicators $\beta_{k}$ measure the regularity of the $k$ th polynomial approximation $\hat{f}^{k}\left(x_{i}\right)$ at the stencil $S_{k}$ and are given by

$$
\beta_{k}=\sum_{l=1}^{2} \Delta x^{2 l-1} \int_{x_{i-\frac{1}{2}}}^{x_{i+\frac{1}{2}}}\left(\frac{d^{l}}{d x^{l}} \hat{f}^{k}(x)\right)^{2} d x .
$$

The expression of the $\beta_{k}$ in terms of the cell averaged values of $f(x), f_{i}$ are given by

$$
\begin{aligned}
& \beta_{0}=\frac{13}{12}\left(f_{i-2}-2 f_{i-1}+f_{i}\right)^{2}+\frac{1}{4}\left(f_{i-2}-4 f_{i-1}+3 f_{i}\right)^{2}, \\
& \beta_{1}=\frac{13}{12}\left(f_{i-1}-2 f_{i}+f_{i+1}\right)^{2}+\frac{1}{4}\left(f_{i-1}-f_{i+1}\right)^{2}, \\
& \beta_{2}=\frac{13}{12}\left(f_{i}-2 f_{i+1}+f_{i+2}\right)^{2}+\frac{1}{4}\left(3 f_{i}-4 f_{i+1}+f_{i+2}\right)^{2} .
\end{aligned}
$$

and

$$
\tau_{5}=\left|\beta_{0}-\beta_{2}\right| .
$$

All $\beta_{k}^{z}$ are smaller than unity and they are all close to 1 at smooth parts of the solution.

The general idea of the weights definition (16) is that on smooth parts of the solution the smoothness indicators $\beta_{k}$ are all small and about the same size, generating weights $\omega_{k}$ that are good approximations to the ideal weights $d_{k}$. On the other hand, if the stencil $S_{k}$ contains a discontinuity, $\beta_{k}$ is $O(1)$ and the corresponding weight $\omega_{k}$ is small relatively to the other weights. This implies that the influence of the polynomial approximation of $h_{i \pm \frac{1}{2}}$ taken across the discontinuity is diminished up to the point where the convex combination (14) is essentially non-oscillatory. Fig. 1 shows the case where stencil $S_{2}$ is discontinuous, yielding $\beta_{0}$ and $\beta_{1}$ to be much smaller than $\beta_{2}$. By (16), this results on $\omega_{2}$ being a small number in the convex combination (14).

The process synthesized by (14)-(15) is called the WENO reconstruction step, for it reconstructs the values of $h(x)$ at the cell boundaries of the interval $I_{i}=\left[x_{i-\frac{1}{2}}, x_{i+\frac{1}{2}}\right]$ from its cell averaged values $f(x)$ in the stencils $\left\{S_{k}, k=0,1,2\right\}$.

Following [1], the hyperbolicity of the Euler equations (29), ignoring the source term $\mathbf{S}$, admits a complete set of right and left eigenvectors for the Jacobian of the system. The eigenvalues and eigenvectors are obtained via the linearized Riemann solver of Roe [21]. The first order Lax-Friedrichs flux is used as the low order building block for the high order reconstruction step of the WENO schemes (see equation (2.5) in [1]). After projecting the fluxes on the characteristic fields via the left eigenvectors, the high order WENO reconstruction step is applied to obtain the high order approximation at the cell boundaries, which are projected back into the physical space via the right eigenvectors. We refer to [1] for further details on the algorithm. 


\section{Particle-mesh Method}

\subsection{Particle solver}

The Lagrangian tracking algorithm of the particles consists of three stages per particle, including searching the element that a particle is located in, interpolating the field variables to the particle location, and pushing the particle forward with a time integration method.

Locating the host cell of a particle is a trivial task on a structured grid. In [14, 17] a tracking algorithm was discussed in a system that only couples the field equations to the particles in one direction, e.g., passive advection. It was shown that interpolation and time integration may, in most cases, be of a lower order than the approximation order of the spatial and time discretization of the field equations to accurately capture the particle traces. Jacobs and Hesthaven [14], however, found that to avoid aliasing errors and an unphysical numerical total energy increase, the order of interpolation has to equal the approximation order $k$ of the stencil $S_{k}$ and the time integration of the particle solver and the carrier phase solver have to match.

We compare lower and higher-order interpolation in this paper. In the section below we discuss several interpolation schemes, including an ENO interpolation that is ideally suited for interpolation of flow solutions containing shock discontinuities. Throughout the paper, we take the scheme used for time-integration the same for the particle and the Euler solver.

\subsection{Coupling of WENO and particle solver}

The particle and the carrier phase exchange momentum and energy. The carrier phase velocity and temperature at the particle position determines the influence of the carrier phase on the particle. This velocity and temperature is determined by interpolation of carrier flow values at the cell centers, $i$, surrounding the particle position. The particle influence is distributed on the carrier phase by the weighing function $\mathbf{K}$ in (11). The interpolation method and weighing function determine the accuracy and characteristics of the Eulerian-Lagrangian frame coupling.

Low-order interpolations are well known to lead to aliasing errors and instability in particle-mesh methods [10]. High-order interpolation and weighing reduces these inaccuracies [10, 20]. We therefore search for the high-order interpolation and weighing that is consistent with the high-order WENO-Z method.

In what follows, we present the lower-order interpolation and weighing for reference, and then discuss high-order central interpolation. Gibbs oscillations plague the accuracy of the central interpolation over shocks. Here, we propose and test an ENO interpolation to improve interpolation in these areas. A highorder spline interpolation [20] reduces aliasing errors and noise in the source determination in (11).

\subsubsection{Interpolation}

In smooth flow areas without shocks, a central interpolation to the particle position is the most accurate and consistent with the finite difference WENO-Z scheme, and thus preferred. We use the Lagrange interpolating polynomial of degree $k$,

$$
\mathbf{P}_{k}\left(x_{p}\right)=\sum_{i=i_{p}-k / 2}^{i_{p}+k / 2} \mathbf{Q}\left(x_{i}\right) l_{i}\left(x_{p}\right),
$$

where $i_{p}$ represents the nearest cell center to the left of the particle position. The number of interpolating points $k$ equals the number of points used in the $k$-th order WENO scheme. In the case of the fifth order WENO scheme, $k=5$.

In shocked regions the centered interpolation will produce undesirable Gibbs oscillations. With an ENO interpolation [18], these oscillations are essentially removed. In ENO interpolation, the interpolating points are determined based on smoothness of the function measured by the divided differences. The $k$-th degree divided differences are determined first. 




Figure 2: One dimensional ENO stencil for interpolation to a particle located near a shock. The interpolation stencil is determined based on the divided differences at the particle's nearest grid point to the left of the particle.

The 0 -th order divided differences of $\mathrm{Q}$ are defined by:

$$
Q\left[x_{i}\right] \equiv Q\left(x_{i}\right) .
$$

The $j$-th degree divided difference for $j \geq 1$ are defined by

$$
Q\left[x_{i}, \cdots, x_{i+j}\right] \equiv \frac{Q\left[x_{i+1}, \cdots, x_{i+j}\right]-Q\left[x_{i}, \cdots, x_{i+j-1}\right]}{x_{i+j}-x_{i}} .
$$

Starting from a two point stencil , $\left\{x_{i_{p}}, x_{i_{p}+1}\right\}$, the interpolation stencil is expanded to $k$ points based on a comparison of the divided differences of the increasing order at $i_{p}$ (See Fig. 2). The smallest second order divided differences at $i_{p}$ of the two potential three point stencils $\min \left\{Q\left[x_{i_{p}-1}, x_{i_{p}}, x_{i_{p}+1}\right], Q\left[x_{i_{p}}, x_{i_{p}+1}, x_{i_{p}+2}\right]\right\}$ indicates the smoothest interpolation stencil and is therefore chosen. This procedure is repeated until a $k$ point interpolant is found. The Lagrange interpolant in (23) then interpolates the relevant gas properties to the particle position.

In Fig. 2, we give an example of a typical ENO stencil close to a shock. We find the nearest grid point to the left of the particle. The magnitude of the first order divided differences at this grid point are larger than the divided differences to the left because of the shock jump. The stencil is therefore extended to the left. The same holds for the divided difference of a higher order than one. So, the ENO stencil will be preferential one-sided to the left of the particle if the particle is located in the cell including a shock.

In two dimensions, the same procedure can be used along the separate dimension on the tensor grid. The divided differences are determined along horizontal and vertical lines in the grid. With the one dimensional approach outlined above, we find the left most and bottom most grid point of the interpolation stencil with $k \times k$ points for each grid point in the domain.

We give an example of a two dimensional ENO stencil in Fig. 3. The particle's nearest grid point is found to the left and bottom of the particle. The left and bottom point of the ENO stencil are determined by comparison of the divided difference along the horizontal and vertical line crossing the nearest grid point. If a particle is located in a cell with a shock, then the ENO is one-sided to the left and bottom of the particle. We note that if two shocks cross the $k$ interpolation stencil, then this procedure will fail to recognize the second shock. We did not encounter stability problems in the simulations and a more sophisticated method based on the high order multi-resolution analysis is under study.

\subsubsection{Weighing}

Low-order particle-methods [10] usually weigh with a zeroth or first order function for $\mathbf{K}(x, y)$ in $(11)$ as shown in Fig. 4. 




Figure 3: Two dimensional ENO stencil for interpolation to a particle located near to a shock. The left and bottom point of the interpolation stencil is determined based on the divided differences along the horizontal grid lines and the vertical grid lines at the particle's nearest grid point to the left and bottom of the particle position.

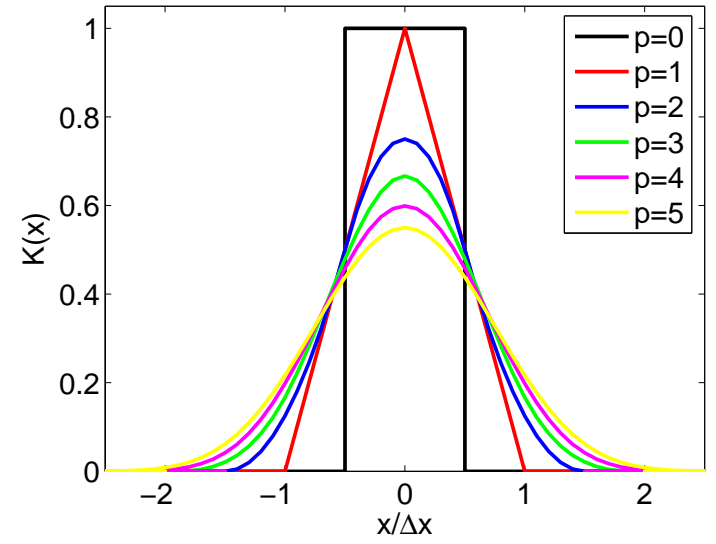

Figure 4: The $p$-th order weighing function. 
The zeroth order weighing function is a tophat function,

$$
\mathbf{K}(x, 0)=\frac{1}{\Delta x}, \quad 0 \leq x \leq \frac{1}{2} \Delta x,
$$

that weighs the particle influence to the nearest grid point. The first order weighing function,

$$
\mathbf{K}(x, 0)=\frac{1}{\Delta x} \frac{\Delta x-x}{\Delta x},
$$

typically used, can be interpreted as a local area weighing. The particle splits a cell into two subcells. The area of the sub-cell to the left determines the particle influence to the grid point on the right of the particle and vice versa. In two dimensions the quadrilateral cell is divided into four new subcells by the particle. The subareas of these are weighted to opposite grid points.

The lower order weighing functions are inconsistent with the higher-order method. The lack of smoothness of the particle shape results in Gibbs type phenomena that affect accuracy and introduce noise in the source term, S. The non-smooth shape is also enhancing instability [10]. Many of these undesirable affects can be reduced by weighing with the shape function described in [19] or weighing with a high order interpolant.

Several options are in principle possible for a higher order weighing. Desirable properties of the weighing function are:

$$
\left\{\begin{array}{l}
\frac{1}{A} \int_{A} \mathbf{K}=1 \\
\max (\mathbf{K}) \text { at } x_{p} \\
M-1 \text { differentiable } M>1 .
\end{array}\right.
$$

The first criterion states that the area $(A)$ weighted average of $\mathbf{K}$ is equal to one and ensures that the magnitude of individual particle momentum and energy is conserved. The second criterion ensures that the particle influence is highest at the the particle location. The third ensures smoothness of the function up to order $M$ consistent with the $M$-th order stencil used for the WENO-Z method.

We consider the high-order spline interpolation discussed in [20], that have all three properties. The spline $S_{k}$ is constructed by the convolution of the square nearest-grid-point or zero order weighing function. For large $k$ the spline approaches the Gaussian function. The zeroth mode of the function in wave space is free of aliasing errors, and the higher component of the function in wave space are smaller than equivalent Lagrangian interpolations.

\section{$5 \quad$ Numerical Experiments}

We test and validate the WENO-Z based PSIC algorithm with one and two dimensional computations of the particle-laden flow that evolves when a shock runs through a cloud of particles. We shall refer the third order WENO-Z method with the second order central interpolation scheme as PSIC/WENO-Z-3 and the fifth order WENO-Z method with the fifth order ENO interpolation scheme as PSIC/WENO-Z-5 or simply PSIC/WENO-Z, unless specified otherwise.

To summarize the algorithm, we approximate the system of hyperbolic Euler equations (2) in the Eulerian frame,

$$
\frac{d \mathbf{Q}}{d t}=-\nabla \cdot \vec{F}(\mathbf{Q})+\mathbf{S}\left(\vec{x}_{p}-\vec{x}\right),
$$

on each grid point. A $N_{p}$ number of particles are individually traced in the Lagrangian frame with

$$
\begin{aligned}
\frac{d \vec{x}_{p}}{d t} & =\vec{v}_{p} \\
\frac{d \vec{v}_{p}}{d t} & =f_{1}\left(\frac{\vec{v}_{f}-\vec{v}_{p}}{\tau_{p}}\right)-\left.\frac{1}{\rho_{p}} \nabla P\right|_{f}, \\
\frac{d T_{p}}{d t} & =\frac{1}{3} \frac{N u}{\operatorname{Pr}}\left(\frac{T_{f}-T_{p}}{\tau_{p}}\right) .
\end{aligned}
$$


Interpolation determines $\vec{v}_{f}$, and $T_{f}$, while weighing determines $\mathbf{S}\left(\vec{x}_{p}-\vec{x}\right)$.

The resulting system of ODE can be updated implicitly or explicitly. If the particle phase and continuum phase have different time scales then an implicit-explicit method can increase the scheme's efficiency [15]. In this study, time integration is performed without typical splitting used for many particle-mesh methods e.g. leap-frog methods [10]. We employed the third order Total Variation Diminishing Runge-Kutta scheme (RK-TVD) [2]:

$$
\begin{aligned}
\vec{U}^{1} & =\vec{U}^{n}+\Delta t L\left(\vec{U}^{n}\right) \\
\vec{U}^{2} & =\frac{1}{4}\left(3 \vec{U}^{n}+\vec{U}^{1}+\Delta t L\left(\vec{U}^{1}\right)\right), \\
\vec{U}^{n+1} & =\frac{1}{3}\left(\vec{U}^{n}+2 \vec{U}^{2}+2 \Delta t L\left(\vec{U}^{2}\right)\right)
\end{aligned}
$$

where $L$ is the spatial operator as in the right side of (29) or the right hand side of (32). The CFL number is set to 0.4 .

The following numerical experiments were performed on a SUN workstation equipped with a AMD Opteron(tm) 250 processor and 3GB of memory.

\subsection{One dimensional shock interacting with a cloud of particles}

We consider the one dimensional problem of a shock running into a cloud of particles, that was studied extensively computationally and experimentally by Boiko et al. [12]. A shock tube problem is setup in the domain $x \in[-5,6]$ with length, $L=11$. The state of the pre-shock flow is

$$
\left[\rho_{R}, u_{R}, p_{R}\right]=[1,0,1] .
$$

The post-shock state can be computed via the well-known Rankine-Hugoniot relations for a given Mach number $M_{s}$. Here, we use $M_{s}=2.8$ with the shock at $x_{s}=0$. A reflective boundary condition is imposed on both ends of the shock tube.

A cloud of particles with a volume concentration of $3 \%$ is initialized in the interval $[0,0.2981]$. The particle response time and density are, $\tau_{p}=3.9296 \times 10^{3}$ and $\rho_{p}=1200$, respectively, corresponding to the experiment with acrylic particles in [12]. We take the Reynolds number needed to compute the particle traces according to the experiment at $R e_{f}=1.7638 \times 10^{6}$.

As a reference, we perform computations with the fifth-order WENO-Z method and particle interpolation and weighing based on a two point stencil. In Fig. 5 we compare the pressure evolution for three grid spacings, $\Delta x=L / N, N=200,400,800$ with the computations performed in [12] with a classical second order PSIC method based on a third-order Rusanov method. It is noted that experiments of the particle-shock flow in a shock tube in [12] compared well with the PSIC computations.

A left moving reflective shock is generated when the shock hits the cloud of particles shortly after time $t>0$. Moreover, an expansion fan is formed at the rear end of the cloud after the shock has passed the cloud of particles. It is clear in Fig. 5 that the high order WENO-Z method gives a sharper representation of the shock and improves the resolution of expansion fan as compared to the Rusanov based method. We underscore the importance of a high order carrier flow solver in Fig. 6 by comparing PSIC based on a third, fifth and seventh order WENO-Z method. The third order PSIC/WENO-Z-3 diffuses the shock and the expansion fan much like the Rusanov based PSIC method, where the higher order PSIC/WENO-Z increasingly sharpens the shock capturing and improves the resolution in the expansion fan.

To fully take advantage of the high-order features of the WENO method the PSIC method and coupling have to be high-order. This requires a high-order interpolation of the carrier flow to the particle position. In smooth regions of the flow, a high-order central interpolation scheme is most accurate, however, in shocked regions Gibbs oscillations can be expected with a central interpolation scheme if the order of the interpolation is greater than one. Figure 7 confirms the inaccurate solution with a central interpolation scheme. The pressure solution oscillates to the left of the right running shock and to the right of the reflected shock. The oscillations persist throughout the computed time interval. 


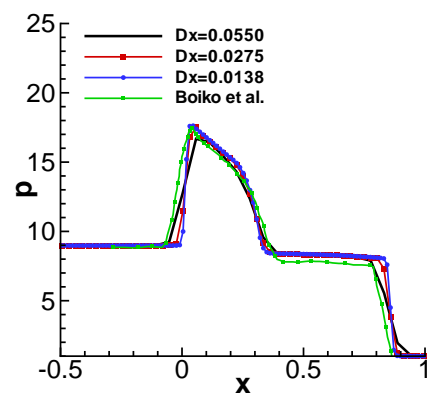

(a) $t_{1}=0.275$



(b) $t_{2}=0.550$



(c) $t_{3}=0.825$

Figure 5: Pressure profile at times (a) $t_{1}=0.275$, (b) $t_{2}=0.55$, and (c) $t_{3}=0.825$ for three grid spacing $\Delta x=0.055,0.275,0.01375$ as computed with the fifth order PSIC/WENO-Z-5 method, compared to the third-order Rusanov based method by Boiko et al. [12].

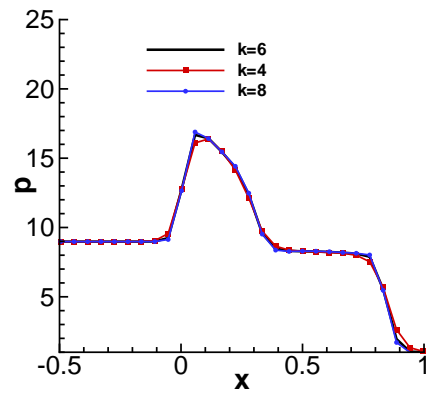

$t_{1}=0.275$

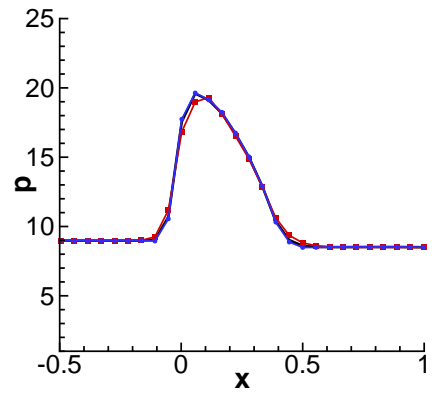

$t_{2}=0.550$

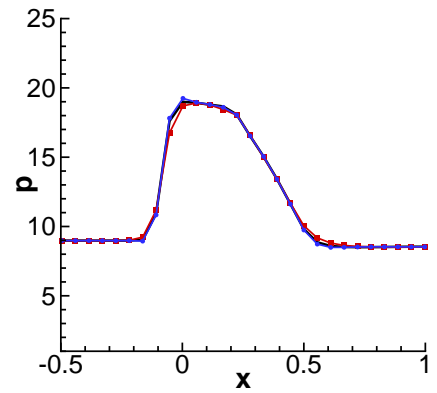

$t_{3}=0.825$

Figure 6: Pressure profile at times (a) $t_{1}=0.275$, (b) $t_{2}=0.55$, and (c) $t_{3}=0.825$ for the third, fifth and seven order PSIC/WENO-Z method.


Figure 7: Pressure profile at times (a) $t_{1}=0.275$, (b) $t_{2}=0.55$, and (c) $t_{3}=0.825$ for the fifth order PSIC/WENO-Z-5 method with interpolation based on a two points, $s=2$, three points, $s=3$, and five points, $s=5$, interpolation stencil. 
With ENO interpolation these oscillations can be prevented (Fig. 8). The pressure trend to the left of the right-running shock now matches well with the solution computed via the low-order interpolation scheme.
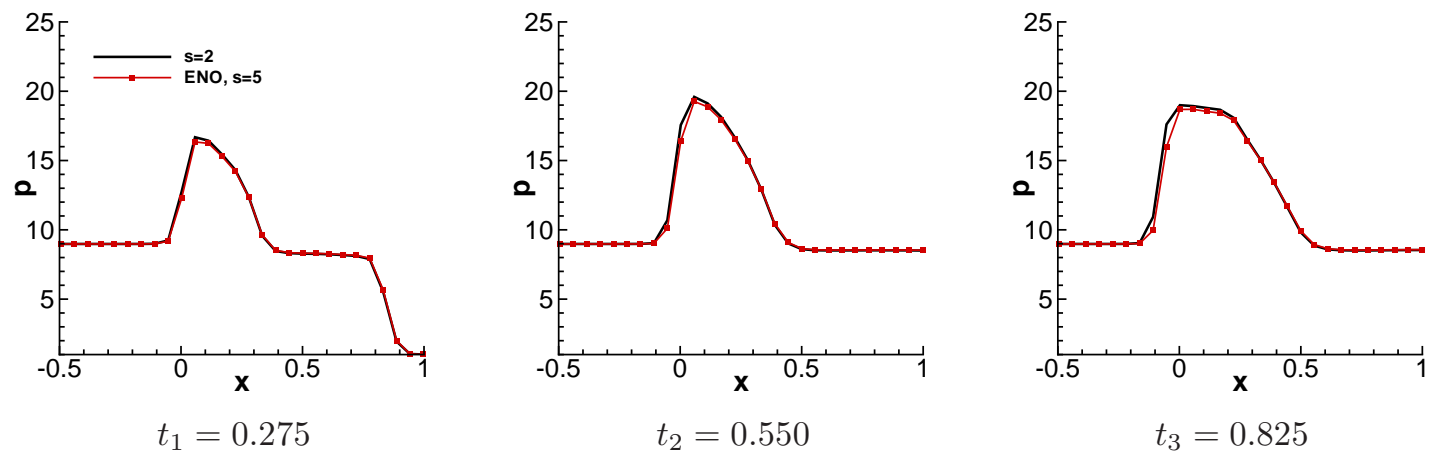

Figure 8: Pressure profile at times (a) $t_{1}=0.275$, (b) $t_{2}=0.55$, and (c) $t_{3}=0.825$ for the fifth order PSIC/WENO-Z-5 method with a two point interpolation stencil, $s=2$, and a five point ENO interpolation stencil, $s=5$.

With increasing order of the weighing function, we observed a slightly smoother source term, $S$, that can be expected to lead to reduced aliasing errors and noise in the coupling between the carrier phase and the particle phase. No significant difference between the solutions of the second order and high order weighing (not shown) were observed for this case. ,

\subsection{Two dimensional shock interaction with a cloud of $40 \mathrm{~K}$ bronze particle}

In a more rigorous test, we study the shock-particle interaction in two dimensions. In Kiselev et al. [13], the two dimensional computation was performed with a PSIC method based on a third order Rusanov scheme. We use their setup to validate the high order WENO-based PSIC. We initialize a right running Mach three shock $M_{s}=3$ at $x_{s}=0.175$ in a rectangular domain $[0,3] \times[-0.611,0.611]$ (see the top figure in Fig. 10). Inflow and outflow boundary conditions are specified, respectively, in the $x$ direction. Periodic boundary conditions are imposed in the $y$ direction. A uniformly distributed bronze particle cloud is seeded in a rectangular shape, $[0.175,0.352] \times[-0.044,0.044]$, with zero initial velocity. The volume concentration of the particles in the cloud is $4 \%$. The particle response time and density are, $\tau_{p}=51.69$ and $\rho_{p}=7.42 \times 10^{4}$, respectively, corresponding to an experiment with the bronze particle cloud in [13]. We take the Reynolds number needed to compute the particle traces according to the experiment at $R e_{f}=3.387 \times 10^{7}$. In our discussion of the results below, we shall refer to the collection of the particles as shape.

In the following simulations, the number of grid points used to solve the Euler equation in the Eulerian frame is $1500 \times 500$ in the $x$ and $y$ directions respectively. The total number of particles is $40 \mathrm{~K}$. The CFL number is 0.4 .

In the two dimensional case, the PSIC/WENO-Z that uses a high order central interpolation scheme is found to be more prone to instability. The Gibbs oscillations introduced by high order central interpolation are not dissipated by the WENO-Z method in two dimensions, resulting in an unstable scheme. The essentially non-oscillatory characteristic of ENO eliminate these numerical oscillations. The ENO computations were stable and were in excellent agreement with published results and low order computations.

Using ENO interpolation scheme, however, comes at the price of evaluating the divided differences and finding the appropriate smooth interpolating stencils for particles located within a cell. In our limited tests with $40 \mathrm{~K}$ particles, approximately $20 \%$ of the total CPU time in the particle interpolation stage is used to locate the smooth interpolation stencils. 
In Fig. 9, a contour plot of the vorticity magnitude $|\omega|$ computed with the third order PSIC/WENO-Z method using a second order central interpolation scheme (PSIC/WENO-Z-3) is compared to the vorticity magnitude contour computed with the fifth order PSIC/WENO-Z method using high order ENO interpolation scheme (PSIC/WENO-Z-5) at the top and bottom figures, respectively. The solution computed by the third order method is far more dissipative than the fifth order scheme; The small vortical roll-up generated by a small jet moving to the right at the symmetry axis $(y=0)$ is clearly under-developed in the third order method. The small scales disturbances generated by the particle-wave interaction throughout the figure are smoothed out as well in the third order method. The recirculation structures right behind the cloud are stronger (higher vorticity) and richer (more detail) in the fifth order solution as compared to the third order solution.
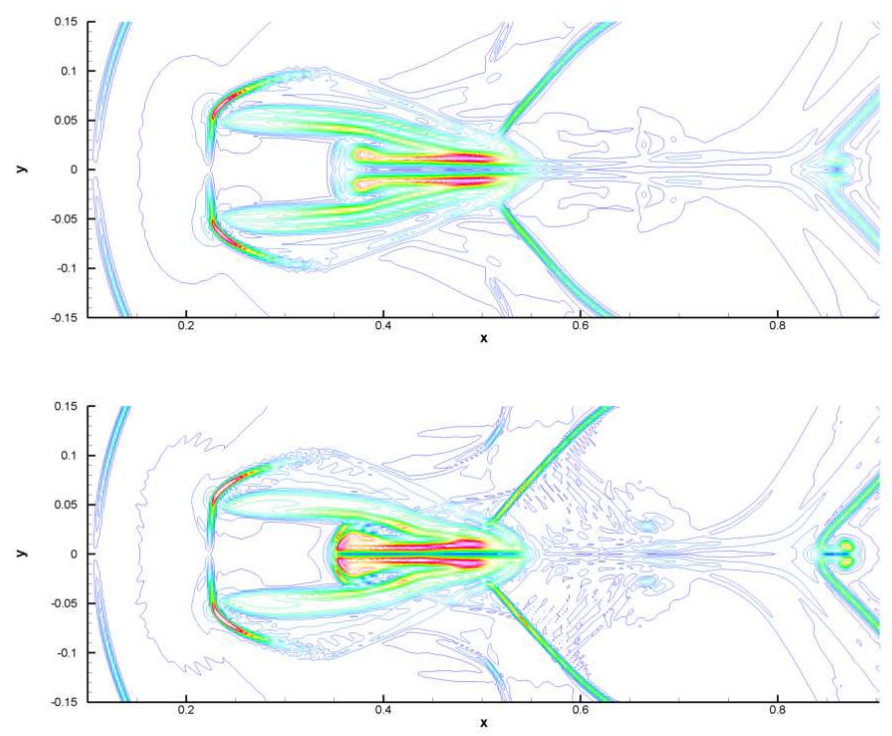

Figure 9: Contour of vorticity magnitude $|\omega|$ of the Mach 3 shock interaction with $40 \mathrm{~K}$ bronze particles as computed by (Top) the third order PSIC/WENO-Z-3 and (Bottom) the fifth order PSIC/WENO-Z-5 methods at time $t=0.225$. The contour lines are between 5 and 305 with an increment of 10 . The number of grid points for the Euler equation in the Eulerian frame is $1500 \times 500$ in the $x$ and $y$ directions respectively.

In the following, we describe the flow evolution of the shock-particle cloud interaction using the fifth order (PSIC/WENO-Z-5) method to illustrate the complexity of this flow, and the potential of these methods to capture detailed multi-scale flow physics for long times.

In Figs. 10-11, we present snapshots of the density $\rho$ (left column), the vorticity magnitude $|\omega|$ (middle column) and the trajectory of the particles (right column) at non-dimensional times $t=0,0.05,0.1,0.225$, 0.5, 0.75. Fig. 12 shows the density, $\rho$, contours with the trajectory of the particles superimposed on it at time $t=1$ for $x \in[0.05,0.205]$. The dotted rectangle in the figures shows the original shape and position of the particles at $t=0$ for reference.

In the time interval from $t=0$ to $t=0.05$, the right running Mach 3 shock hits the particle and a reflected bow shock forms at the front end of the shape as seen in the density plot (Fig. 10, left column). The right running shocks move along the top and bottom sides of the shape and are curved close to the shape. Strong vorticity $\omega$ is generated by the accelerated flow in the wake of the curved shock. The shear layer emanating from the front corners of the shape are also clearly visible by the increased vorticity magnitude in Fig. 10 (middle column). The accelerated flow stagnates at the front of the shape and compresses the shape. The particles at the front end move towards the right at this early time and increase the particle density 
(Fig. 10, right column).

At times $t=0.1$ and $t=0.225$, the main shock has moved past the shape and the two curved shocks originated from the top and bottom of the shape have moved towards and crossed the symmetry line at $y=0$. A Mach reflection occurs once the curved shocks have crossed the symmetry lines, that connects the curved shock and the right running main shock. Further in time, the flow complexity significantly increases, when the non-linearly interacting curved shocks affect the compression wave in the particle cloud, and the vorticity generated at the rear end of the cloud.

The force exerted by the particles on the accelerated gas behind the main shock leads to a flow pattern comparable to a flow over a blunt body. The flow separated on the top and bottom sides of the shape, and a recirculation forms at the back of the shape. The shape is compressed inward on the top and bottom sides toward the symmetry line at $y=0$ as shown in the trajectory of the particles. The strong vorticity in the separated shear zones pulls the particles out of the shape, which leads to the formation of the particle arms and legs on the side and the back of the shape. The arms and legs follow the separated shear layers. At the back of the shape, the particle legs thus bend inward and the particle arm on the sides moves upward and downstream.

The gas density increases inside the shape, but the pressure remains constant (not shown). The particles cool the gas through heat exchange [13]. The lowered temperature leads to an increased density to satisfy the ideal gas law (see Fig. 10), since the pressure remains constant in the cloud to balance the particle force.

The overall flow field maintains its symmetry up to this point in time.

The flow becomes unstable and loses its symmetry at later time (See figures for $t=0.5$ and $t=0.75$ ). In the wake of the shape an asymmetric shedding is observed in the density and vorticity contour (Fig. 11, left two columns). Increasingly more particles are dispersed upward and downstream of the cloud forming long and thicker particle arms into the main flow and around the shape. A compression wave that formed at the side of the shape at early time, has now moved sideways away from the symmetry line, and has run into the particle arms. This compression wave reshocks the particle-laden flow in the arms, and further widens the particle arms. This re-shocking induces intermittent wave patterns in the side shear layer that interact with the particles forming streaks and bunching of the particles.

Throughout the computed time interval the side arms shield the high-speed flow generated by the right running shock from the wake of the shape. Only a small number of particles are dispersed in the legs at the rear of the shape, since the side arms divert the main high speed flow away from the shape and the relatively low flow velocities in the wake of the shape do not carry the particles as much as in the side arm.

In Fig. 12 the density flow field with a superimposed particles trajectory at $t=1$ shows the flow field in a larger domain, and illustrate the tremendous complexity of this flow, with coupled multiple shock reflections, and particle dispersion. A full length animation of the density field of the Mach 3 shock-particle-laden flow is posted at [http://attila.sdsu.edu/ jacobs/PICTURES/Rho_40K.avi].

\section{Conclusions and Future Developments}

We have developed a high order particle-source-in-cell (PSIC/WENO-Z) algorithm based characteristic based conservative finite difference WENO-Z method for computation of particle-laden flows with shocks.

A high order ENO interpolation ensures a stable high order interpolation of the carrier gas to the particle position. In one dimension, the interpolation stencil is determined using divided differences. A Lagrange interpolant then interpolates the carrier gas from the finite difference grid to the particle position. In two dimensions, the divided differences along horizontal and vertical lines on the tensor grid determine the interpolating stencil. A Lagrange interpolation again determines the carrier gas properties at the particle location.

High order central interpolation was shown to be inaccurate and in two-dimensional cases unstable. A high order weighing function, that reduces aliasing errors and noise, was implemented. A third order RK-TVD scheme integrates both the particles and carrier gas phase in time.

We tested the algorithm in a one dimensional shock-particle-cloud interaction problem. We showed 

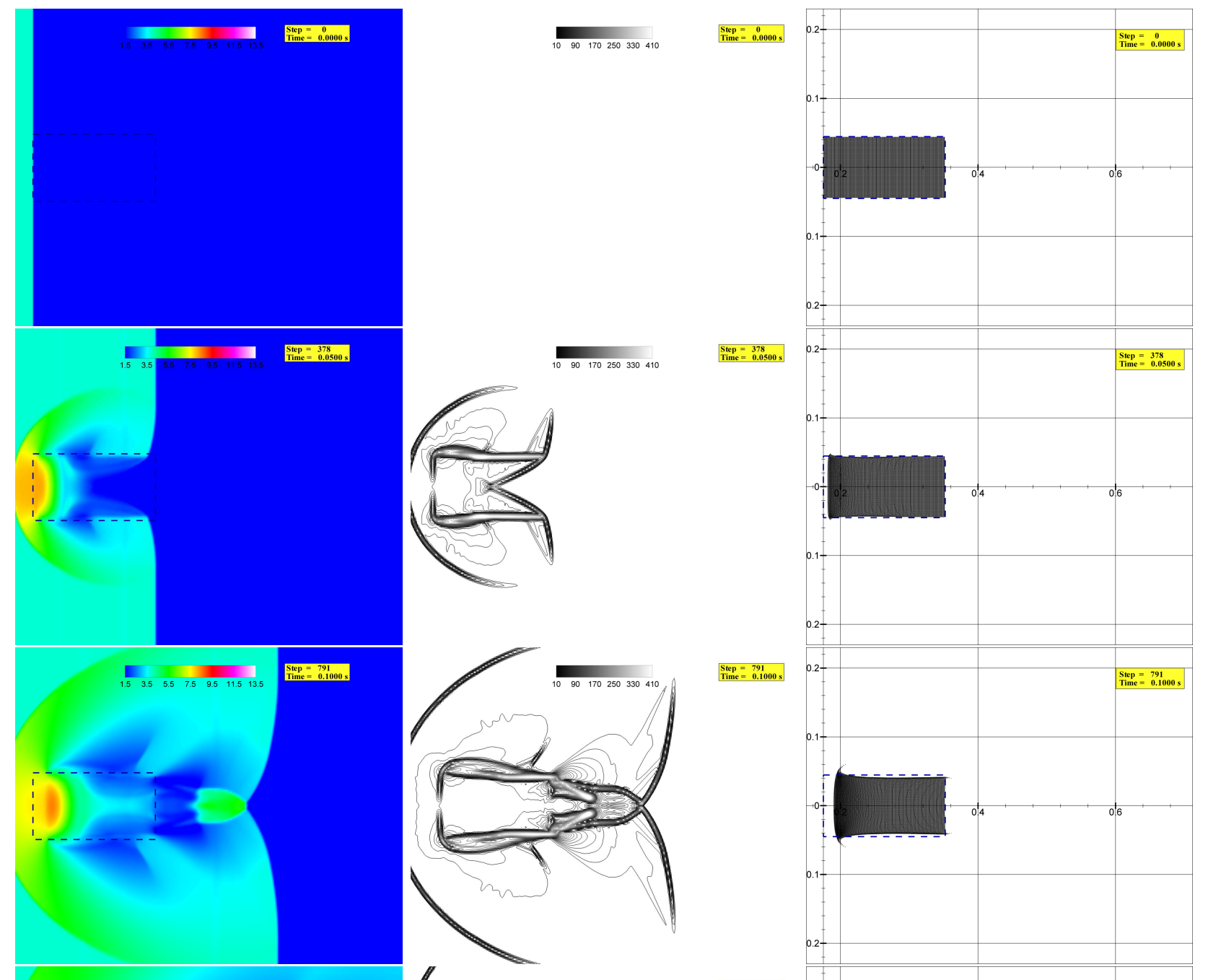

Step $=791 \mathrm{~s}$
Time $=0.1000 \mathrm{~s}$

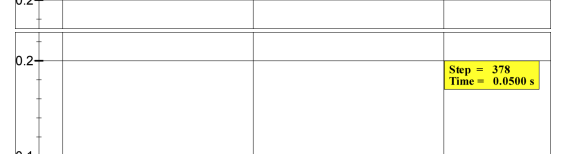



Vorticity $|\omega|$



Particle's Trajectory

Figure 10: A snapshot of the density $\rho$ (left column), the vorticity magnitude $|\omega|$ (middle column) and the trajectory of the particle clouds (right column) for time $t=0,0.05,0.1,0.225,0.5,0.75$ (from top to bottom) as computed by the fifth order PSIC/WENO-Z-5 method with ENO interpolation scheme. The dotted rectangle in the figures showed the original shape and position of the particle clouds at $t=0$ for easy reference. The shock Mach number is $M_{s}=3$. The number of grid points used in the Eulerian frame is $1500 \times 500$ in the $x$ and $y$ directions respectively. The total number of bronze particle clouds is $40 \mathrm{~K}$. 




Density $\rho$



Vorticity $|\omega|$

Particle's Trajectory

Figure 11: see figure 10 for caption.

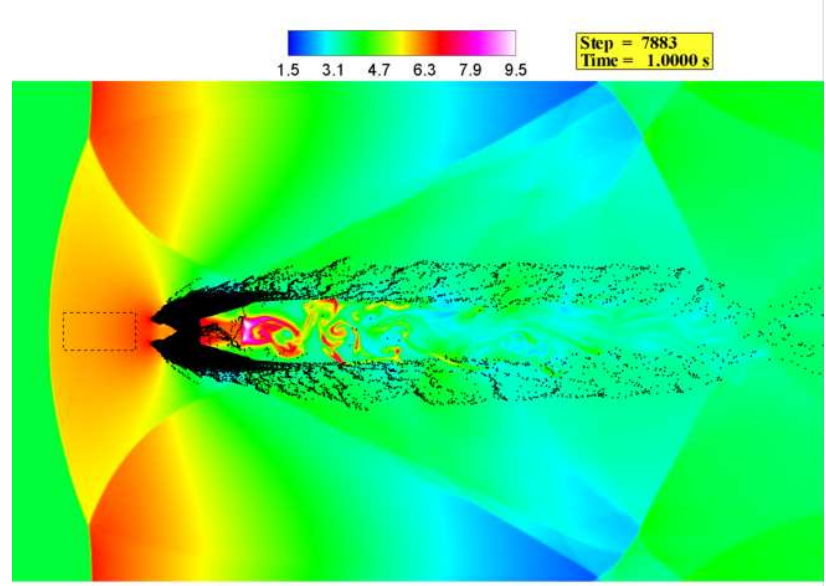

Figure 12: The density $\rho$ contour and the superimposed trajectory of the particle clouds at time $t=1$ as computed by the fifth order PSIC/WENO-Z-5 method with ENO interpolation scheme. The domain size in $x$ is $[0.05,2.05]$. See figure 10 for additional caption. 
that the high-order WENO based PSIC/WENO-Z method captures shock movement more accurately than the third order Rusanov method, while the particle motion and the expansion region behind the shock compared well to published results. Numerical induced oscillations were clearly visible when a central high order particle interpolation was used. The central high order weighing slightly smoothened the solution, but did not have a significant effect on the particle-laden flow.

A two dimensional test on a shock-particle-cloud interaction, confirmed the above findings. In two dimensions, the high-order central interpolation is unstable. Long time integration illustrated that the algorithm is capable of capturing shocks and fine scale flow features at the same time.

Our current efforts focus on a one sided weighing of the particles consistent with the ENO interpolation. The central high order weighing we employed in this study diffuses the particle influence over the grid. Even though this leads to favorable stability properties, the smearing at shock locations is likely to negatively affect the accurate capturing of the shock capturing.

We are also working on a particle-source-in-cell method with a hybrid spectral-WENO kernel in a multi-domain framework for solution of the gas dynamics. In the hybrid method [22], the WENO method effectively captures shocks in shocked subdomains, while in smooth subdomains, a more efficient spectral method solves the gas flow. A high order smoothness indicator based on the high order multi-resolution analysis switches the WENO and spectral methods adaptively in space and time as the flow evolves in time in each subdomain. A consistent high-order particle interpolation and weighing in the hybrid is currently under development.

We illustrated the potential of this method to identify detailed flow physics that are found in high-speeds flow environments. In future work, we will extend the capabilities of the algorithm to include viscous flow models, large-eddy modeling, chemical reactions, and extension and parallel implementation of the algorithm to three dimension in complex geometry, enabling the analysis of high-speed combustors.

\section{Acknowledgments}

The first author gratefully acknowledges the support of this work in the form of a seed grant by the University Grants Program at San Diego State University. The second author Don gratefully acknowledges the support of this work by the DOE under contract number DE-FG02-98ER25346 and the AFOSR under contract number FA9550-05-1-0123. He also extends his gratitude to the Department of Aerospace Engineering at San Diego State University for hosting his visit.

\section{$\underline{\text { References }}$}

[1] D. Balsara and C. W. Shu, Monotonicity preserving weighted essentially non-oscillatory schemes with increasingly high order of accuracy, J. Comp. Phys. 160, pp. 405-452 (2000)

[2] G. S. Jiang and C. W. Shu, Efficient Implementation of Weighted ENO Schemes, J. Comp. Phys. 126, pp. 202-228 (1996)

[3] A. Harten, B. Engquist, S. Osher and S. Chakravarthy, Uniformly high order essentially non-oscillatory schemes, III, J. Comp. Phys. 71, pp. 231-303 (1987)

[4] C. W. Shu and S. Osher, Efficient implementation of essentially non-oscillatory shock-capturing schemes, J. Comp. Phys. 77, pp. 439-471 (1988)

[5] C. W. Shu and S. Osher, Efficient implementation of essentially non-oscillatory shock capturing schemes, II, J. Comp. Phys. 83, pp. 32-78 (1989)

[6] X. Liu, S. Osher and T. Chan, Weighted essentially non-oscillatory schemes, J. Comp. Phys. 115, pp. 200-212 (1994) 
[7] A. K. Henrick, T. D. Aslam and J. M. Powers, Mapped weighted essentially non-oscillatory schemes: Achieving optimal order near critical points, J. Comp. Phys. 207, pp. 542-567 (2005)

[8] R. Borges, M. Carmona, B. Costa and W. S. Don, An improved weighted essentially non-oscillatory scheme for hyperbolic conservation laws, J. Comp. Phys. 227 No. 6, pp. 3101-3211 (2008)

[9] C. T. Crowe, M. P. Sharma and D. E. Stock, The Particle-Source in Cell (PSI-Cell) Model for GasDroplet Flows, J. Fluids Eng., 6, pp. 325-332, (1977).

[10] C. K. Birdsall and A. B. Langdon, Plasma physics via computer simulation. McGraw-Hill, Inc., 1985.

[11] C. T. Crowe, M. Sommerfeld and Y. Tsuji. Multiphase Flows with Droplets and Particles, Press LLC, Boca Raton, FL (1998).

[12] V. Boiko, V. P. Kiselev, S. P. Kiselev, A. Papyrin, S. Poplavsky and V. Fomin, Shock wave interaction with a cloud of particles, Shock Waves 7, pp. 275-285 (1997)

[13] V. P. Kiselev, S. P. Kiselev and E. V. Vorozhtsov, Interaction of a shock wave with a particle cloud of finite size, Shock Waves 16, 2006, pp. 53-64 (2006)

[14] G. B. Jacobs and J. S. Hesthaven, High-order nodal discontinuous Galerkin particle-in-cell method on unstructured grids, J. Comp. Phys. 214, pp. 96-121 (2006)

[15] G. B. Jacobs, G. Lapenta and J. S. Hesthaven, Simulations of Plasmas with a High-Order Discontinuous Galerkin Particle-In-Cell Solver, AIAA Paper 2006-1171, (2006).

[16] M. Latini, O. Schilling and W. S. Don, Effects of order of WENO flux reconstruction and spatial resolution on re-shocked two-dimensional Richtmyer-Meshkov instability, J. Comp. Phys. 221 No. 2, pp. 805-836 (2007)

[17] G. B. Jacobs, D. A. Kopriva and F. Mashayek, Towards efficient tracking of inertial particles with high-order multidomain methods, J. Comp. Appl. Math. 206, pp. 392-408 (2007)

[18] C. W. Shu., Essentially non-oscillatory and weighted essentially non-oscillatory schemes for hyperbolic conservation laws, ICASE Report 97-65, NASA Langley Research Center, Hampton, VA (1997)

[19] M. R. Maxey, B. K. Patel and L. P. Wang, Simulations of dispersed turbulent multiphase flow, Fluid Dyn. Res. 20, pp. 143-156( 1997).

[20] H. Abe, S. Natsuhiko and R. Itatani, High-order spline interpolations in the particle simulation, J. Comp. Phys. 63, pp. 247-267 (1986)

[21] P. L. Roe, Approximation Riemann solvers, parameter vectors, and difference schemes, J. Comp. Phys. 43, pp. 357 (1981)

[22] B . Costa, W. S. Don, D. Gottlieb and R. Sendersky, Two-Dimensional Multi-Domain Hybrid SpectralWENO Methods for Conservation Laws, Comm. in Comp. Phys., 1, pp. 550-577 (2006) 